Article

\title{
Synthesis, Curing Behavior and Swell Tests of pH-Responsive Coatings from Acryl-Terminated Oligo( $\beta$-Amino Esters)
}

\author{
Krister Hammarling ${ }^{1, *} \mathbb{C}$, Mats Sandberg ${ }^{1,2} \mathbb{C D}^{\mathbb{D}}$, Magnus Engholm ${ }^{1}$, Henrik Andersson ${ }^{1}$ (D) \\ and Hans-Erik Nilsson 1 (iD) \\ 1 Department of Electronics Design, Mid Sweden University, Holmgatan 10, 85170 Sundsvall, Sweden; \\ mats.sandberg@ri.se (M.S.); magnus.engholm@miun.se (M.E.); henrik.andersson@miun.se (H.A.); \\ hans-erik.nilsson@miun.se (H.-E.N.) \\ 2 RISE Acreo AB, Bredgatan 33, 60117 Norrköping, Sweden \\ * Correspondence: krister.hammarling@miun.se; Tel.: +46-010-1428717
}

Received: 19 January 2018; Accepted: 22 February 2018; Published: 23 February 2018

\begin{abstract}
The ability of acryl-terminated oligo( $\beta$-amino esters) (AOBAE) to be coated on fibers and printed electronics without solvents and to be cross-linked to a $\mathrm{pH}$-responsive coatings, makes AOBAE-based coatings a potential type of $\mathrm{pH}$-sensor coating. However, there are currently no reports of AOBAEs used as a $\mathrm{pH}$-responsive coating material in sensor applications. Here we present an investigation of the synthesis, curing behavior and swell tests of AOBAEs. AOBAEs were synthesized from reacting an excess of asymmetric diacrylates with piperazine without the use of any solvents. They were then cross-linked to an insoluble network by UV-curing. Nuclear magnetic resonance (NMR) and Fourier transform infrared (FTIR) spectroscopy were used to characterize the AOBAEs. NMR was used to clarify the irregular structure of the AOBAE. FTIR was used to monitor the effects of UV-curing dose and air exposure on monomer conversion during curing. An interferometric technique was used to monitor the swelling behavior of the coating in response to $\mathrm{pH}$ variations. Swell experiments showed that the AOBAE also responded to $\mathrm{pH}$ variations after polymerization. Therefore, AOBAE is an interesting class of material with potential use as a $\mathrm{pH}$ responsive coating in optical-and printed electronics $\mathrm{pH}$-sensors applications.
\end{abstract}

Keywords: oligo( $\beta$-amino esters); acryl-terminated; 1,3-butanediol diacrylate; piperazine; $\mathrm{pH}$; sensor; coating; thin film

\section{Introduction}

Sensor materials, in the form of coatings on optical fibers or on printed electrodes, opens the possibility for many interesting sensor applications. Optical fiber sensors enable distributed sensing along a fiber at desired points over long distances or in confined spaces. Printed sensors on flexible substrates, such as paper or plastics can, for example, offer production of low-cost sensor devices for point of care applications, construction applications, food packaging and environmental monitoring [1-4].

The demand for $\mathrm{pH}$ sensors is growing, especially in applications such as chemical instrumentation, industry, process control, medical analyses and environmental monitoring. In the field of environmental monitoring there is a special need for distributed sensors to cover large areas [5-7], either as distributed sensors or sensors spread out in numerous locations in for example forests, lakes or landfills. This makes $\mathrm{pH}$ responsive coatings that are compatible with the optical or printed sensor technology particularly interesting. Richter et al. [8] describe two basic principles for transducers based on responsive hydrogels: (a) materials based on mechanical work performed by swelling and 
shrinking and (b) materials that exhibit a change in properties (e.g., densities, mass, volume, stiffness). For both principles, a pH responsive coating in its cured form must be able to change its mechanical-, optical- or electrical parameters in response to $\mathrm{pH}$ variations. Deposition of a material by coating or printing requires a suitable viscosity, wetting on the substrate and the ability to cure the polymer to a solid coating or a printed pattern [9]. Furthermore, deposition of materials to form coatings on optical fibers is preferably carried out without the use of solvents. Therefore, the precursor forming the responsive coating should be a fluid in its neat form under ambient conditions. Since the material is to form a part of a hydrogel-based sensor, cross-linking during curing must be complete and not result in soluble residues. Otherwise, incomplete curing would result in a gradual leak of soluble material from the gel. For this reason, it is important to establish the right conditions for sufficient curing.

Polymerizable molecules containing amino groups would be suitable starting materials for $\mathrm{pH}$-responsive coatings. We have identified coupling of amines with acryalates to ( $\beta$-amino esters) as a viable synthetic path to this type of molecules. More specifically, we have explored the stochiometric balance of diamines and diacrylates to produce oligo( $\beta$-amino esters) with acrylic terminal groups with controlled molecular weight. However, there have to our knowledge been no reports about any investigation on AOBAEs used as a coating material. The AOBAEs are closely related to the poly( $\beta$-amino esters) (PBAEs), which were introduced by Lynn and Langer [10] as gene transfer vector materials, that have been extensively explored for its $\mathrm{pH}$-responsiveness. PBAEs are readily obtained by reacting equimolar amounts of diamines with diacrylates. Coupling of primary amines with acrylates requires heating and once a secondary ( $\beta$-amino ester) is formed, the secondary amino group readily reacts with another acrylate to form a PBAE of tertiary amino ester. The obtained PBAEs are bio-compatible, have low toxicity and are suitable for use in vivo and in environmental monitoring [11]. For example, PBAEs obtained from 1,4-butanediol diacrylate (1,4-BDDA) and piperazine (PIP) have previously been studied as $\mathrm{pH}$ responsive polymers [12]. However, initial tests with acryl-terminated oligomers obtained from reacting 1,4-BDDA and PIP resulted in solid and crystalline materials in room temperature and are hence not suitable for the process of coating of optical fibers. Synthesis of non-crystalline AOBAEs that are fluid at ambient temperatures are therefore needed to produce $\mathrm{pH}$-responsive optical fiber coatings. The crystallinity of polymers depends on the symmetry properties of the polymer structural units. Polymers with symmetric structural units typically have a higher tendency to crystallize than polymers with asymmetric structural units. AOBAEs prepared from 1,4-BDDA and piperazine have symmetric structural units, which may contribute to their observed and in this case undesired, tendency to form crystalline solids. We have therefore explored AOBAEs with asymmetric structural units. By preparing AOBAE from piperazine and 1,3-BDDA we incorporate the asymmetric 1,3-butylene group in the AOBAE. The 1,3-butylene group have a chiral carbon atom, which introduces chirality, as well as a directional "head-to-tail" isomerism due to the un-equivalence of the 1 and 3 positions. It is well known that both tacticity, the arrangement of chiral atoms in a polymer and directional isomerism influence crystallinity in polymers $[13,14]$. The coupling reaction forming ( $\beta$-amino esters) is likely to produce polymers that are atactic and with an irregular directional arrangement of 1,3-butylene groups. AOBAEs synthesized from 1,3-BDDA are therefore more likely to produce non-crystalline materials, which is the aim of the present investigation.

A class of $\mathrm{pH}$ sensitive AOBAEs not previously studied have been examined. The aim is to use the AOBAEs as a coating material, compatible with optical- and printed electronics sensor technology for various applications. Cross-linked AOBAE coatings were obtained by polymerization of AOBAE in the presence of a photo-initiator on substrates pretreated with primers to enhance the adhesion. Curing parameters, such as the irradiation dose and atmospheric conditions (air or inert gas) were investigated, as well as the effect of adhesion promoting primers. NMR was used for molecular structural characterization of AOBAE. FTIR spectroscopy was used to monitor the effect of curing parameters. The $\mathrm{pH}$ responsivity of cured AOBAE coatings were investigated by monitoring the film thickness change in response to $\mathrm{pH}$ variation by using an interferometric measurement technique. The swell responsivity indicates also that the permittivity and the RI will change. Even a 
small change of swelling can have a large impact on a materials permittivity and RI. For example Bolthauser et al. [15] built a humidity sensor in which they measured the effective dielectric constant change in polyimide (polyimide absorbs $1.2 \mathrm{wt} \%$ water [16]). And Dubendorfer et al. [17] reached a $\mathrm{pH}$ resolution of $\pm 1.1 \times 10^{-4}$ when measuring $\mathrm{RI}$ changes for a hydrogel that swelled $35 \% / \mathrm{pH}$ unit. Therefore we believe that the AOBAE can be an interesting candidate as a $\mathrm{pH}$ sensitive coating material for the development $\mathrm{pH}$ sensors using optical- or printed electronics technology.

\section{Materials and Methods}

\subsection{Materials}

1,3-butanediol diacrylate (1,3-BDDA), piperazine (PIP), diphenyl(2,4,6-trimethylbenzoyl) phosphine oxide (TPO) and 3-(trimethoxysilyl) propyl acrylate were purchased from Merck and used without further purification.

\subsection{Instruments and Measurement Setups}

The viscosity measurements were made with Mettler RM180 rheomat at $21^{\circ} \mathrm{C}$ (Mettler Toledo, Columbus, OH, USA) and the testing speeds used were between 50 and $1000 \mathrm{rpm}$. FTIR measurements were obtained using a NicoletTM 6700 FTIR (Nicolet Instrument Technologies Inc., Madison, WI, USA) with a DTGS KBr detector (Nicolet Instrument Technologies Inc., Madison, WI, USA) and a Smart Orbit accessory. For the FTIR measurements, curing was made directly on the Smart Orbit diamond by using a $365 \mathrm{~nm}$ fiber coupled UV-LED (M365F1, Thorlabs, Newton, MA, USA) and a $600 \mu \mathrm{m}$ Ocean Optic patchcord (QP600-2-SR-BX, Ocean Optics inc., Largo, FL, USA). Three different intensities were used for the curing: 2.9, 5.8 and $11.9 \mathrm{~mW} / \mathrm{cm}^{2}$, which were measured using a photodiode power sensor (S120VC) and readout unit (PM100D) from Thorlabs. Film thicknesses in the FTIR experiments were measured after curing with a caliper, Mahr Millitast 1083 (Mahr GmbH, Göttingen, Germany). ${ }^{1} \mathrm{H}$ NMR spectra were recorded with a Bruker Ultrashield $500 \mathrm{MHz}$ NMR spectrometer. For the NMR measurements, the AOBAE was dissolved in deuterated chloroform $\mathrm{CDCl}_{3}$. The swell experiments were performed with an interferometric thin film measurement setup, consisting of an Avantes Avaspec-2048 optical spectrum analyzer (Avantes, Apeldoorn, The Netherlands), Ocean Optics HL-2000-FHSA light source, Ocean Optics QR450-7-XSR reflection probe (Ocean Optics inc., Largo, FL, USA) and Avantes AvaSoft-ThinFilm software (Avantes, Apeldoorn, The Netherlands) to calculate the thickness of the film. The used $\mathrm{pH}$ buffer solutions were controlled by using a Metrohm $632 \mathrm{pH}-\mathrm{meter}$ prior to measurements.

\section{3. $A O B A E$}

Batches of 1,3-BDDA:PIP with a mole ratio of 2:1 were made by dissolving PIP into 1,3-BDDA during stirring at $60^{\circ} \mathrm{C}$, without the use of any solvents. Stirring continued until there were no visible PIP crystals in the solution and then for another $30 \mathrm{~min}$, making it a total of $120 \mathrm{~min}$. The solution was then left to cool to room temperature before further processing. Figure 1 shows the principle synthesis of 1,3-BDDA:PIP with a mole ratio of 2:1. The viscosity of 1,3-BDDA:PIP with a mole ratio of 2:1 was measured to $770 \mathrm{cP}$. 
<smiles>C=CC(=O)OCCC(C)OC(=O)C=CC(=O)OCCC(C)OC(=O)C(=C)C</smiles>

Figure 1. Synthesis of 1,3-butanediol diacrylate (1,3-BDDA) and piperazine (PIP). The diamine nitrogen breaks the double bond on the diacrylate and forms a secondary ( $\beta$-amino ester).

\subsection{FTIR and NMR}

For the FTIR measurements, we put a drop of AOBAE directly on top of the Smart Orbit crystal and the film thicknesses were measured after curing with a $\mu \mathrm{m}$ caliper. The AOBAE drops provided film thicknesses between 200 and $400 \mu \mathrm{m}$. The Smart Orbit accessory uses a reflective measurement method and can therefore only measure a few $\mu \mathrm{m}$ into the sample. Because of the relative thick films used, we ensured that we only measured on AOBAE. The resolution was set to $2 \mathrm{~cm}^{-1}$ and an average of 32 sample spectrums were used. For measurements in inert atmosphere, a dark box was built around the Smart Orbit diamond that was flooded with $\mathrm{N}_{2}$ gas and purged for a minimum of 15 min before the measurements. The box was also used during tests in ambient air atmosphere, but without $\mathrm{N}_{2}$ flowing. Prior to the FTIR measurements, 2.5 or $1 \mathrm{wt} \%$ of TPO was mixed into the AOBAE solution. The estimated conversion error is $\pm 3 \%$, calculated from three batches of 1,3-AOBAE with $2.5 \mathrm{wt} \%$ of TPO.

To establish the relation of power output from the UV-led and curing of the 1,3-AOBAE, intensities of 2.9, 5.8 and $11.9 \mathrm{~mW} / \mathrm{cm}^{2}$ were evaluated in both air and $\mathrm{N}_{2}$. The curing experiments were conducted on a $200-400 \mu \mathrm{m}$ thick film with 2.5 or $1 \mathrm{wt} \%$ of (TPO). The degree of conversion (x) is directly related to the decrease of the IR absorbance and was calculated from Equation (1)

$$
x(\%)=\frac{A_{0}-A_{t}}{A_{0}} \times 100
$$

where $A_{0}$ and $A_{t}$ represent the absorbance peak of the IR band [18,19].

\subsection{Surface Priming of Si-Wafers}

A standard Silicon ( $\mathrm{Si}$ ) wafer was used as a substrate for the swell experiments. The adhesive strength between the coated layer and the substrate was improved by tethering acryl groups to the surface. 3-(trimethoxysilyl) propyl acrylate was spin coated on the substrates at $3000 \mathrm{rpm}$ and left to dry at room temperature before the AOBAE coating was applied.

\subsection{Spin Coating and Curing on Si-Wafers}

Before AOBAE coating and curing on Si-wafers was made, $2.5 \mathrm{wt} \%$ of TPO was dissolved into the AOBAE. 1,3-AOBAE was then spin-coated onto the Si-wafers at $9000 \mathrm{rpm}$ in air atmosphere (for 45, 90 and $135 \mathrm{~s}$ ), forming thin films between 1.4 and $12 \mu \mathrm{m}$. The Si-wafer was then placed in a box that was flooded with $\mathrm{N}_{2}$ for 15 min before curing. 


\subsection{Swell Experiments}

The reflection probe was held at approximately $2 \mathrm{~mm}$ from the Si-wafer surface and a buffer solution (Britton-Robinson buffer [20]) with different $\mathrm{pH}$ was placed between the probe and the coated Si-wafer. The drop filled the whole space between the probe and the Si-wafer, thus there were no disturbing light reflections from the surface of the $\mathrm{pH}$ solution. The 1,3-AOBAE film thickness was first measured in its dry state and used as reference.

\section{Results}

Based on initial experiments, we found that mole ratio 1,3-BDDA:PIP of 2:1 gave an acceptable trade-off between viscosity of the neat AOBAE swelling response and stability to swelling for this study. The composition was then used for further evaluation. 1,3-AOBAE with mole ratio 2:1 is henceforward called 1,3-AOBAE.

\subsection{NMR}

Figure 2 shows the ${ }^{1} \mathrm{H}-\mathrm{NMR}$ spectrum of 1,3-AOBAE with peak assignments indicated. The signals of the acrylic groups are located in the range 5.75-6.4 ppm $(\mathrm{i}, \mathrm{j}, \mathrm{h})$. The major signal at $2.45 \mathrm{ppm}$ is assigned to the protons in the PIP ring and the ethylene groups connecting the amino group with the ester bond $(a, b, c)$. The protons of the 1,3-butylene group are assigned as follows: The signals located around $5 \mathrm{ppm}$ are assigned to the methine proton (f). The two signals around 1.20 and $1.26 \mathrm{ppm}$ are assigned to the methyl group $(\mathrm{g})$. The signal around $4.1 \mathrm{ppm}$ is assigned to the methylene protons adjacent to the ester bond (d) and the signal at $1.82 \mathrm{ppm}$ is assigned to the methylene protons in the central atom of the propylene chain (e). The asymmetric structure of the 1,3-butylene group is not only reflected by the absence of crystallinity in 1,3-AOBAE, but also in the NMR-spectrum. The presence of directional isomerism is indicated in the molecular structure in Figure 2, with either the 1 or 3 position connected to the piperazine unit and by the prime symbol annotations. The chirality of the methine carbons, $\mathrm{f}$ and $\mathrm{f}^{\prime}$, also contributes to the complex NMR-signal patterns of the 1,3-butylene group.

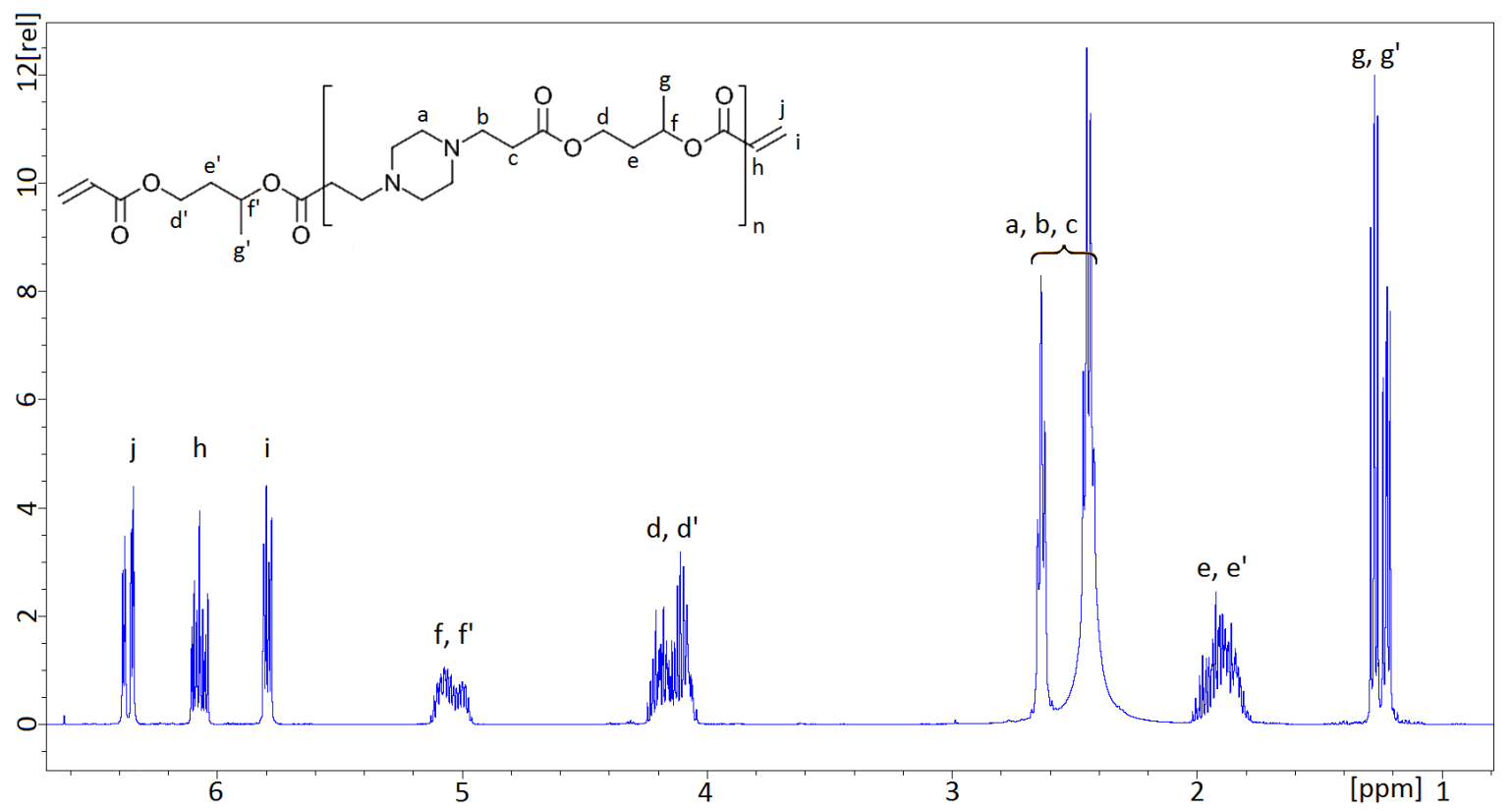

Figure 2. Nuclear magnetic resonance (NMR) spectra of 1,3-AOBAE. a-c are protons in the PIP ring and of the ethylene groups connecting the amino group with the ester bond; $d$ are the methylene protons adjacent to the ester bond; e are the methylene protons in the central atom of the propylene chain; $f$ is the methine proton; $\mathrm{i}, \mathrm{j}$ and $\mathrm{h}$ are the acrylic groups. 


\subsection{FTIR Peak Assignment and Monomer Conversion}

Figure 3 shows the FTIR spectra of 1,3-AOBAE before irradiation, after $5 \mathrm{~s}$ and $480 \mathrm{~s}$ of UV-irradiation under nitrogen atmosphere. The spectral peak assignments were made as follows: (a) $2948 \mathrm{~cm}^{-1} \mathrm{CH} 2$ stretch; (b) $2811 \mathrm{~cm}^{-1} \mathrm{C}=\mathrm{O}$ overtone from $1408 \mathrm{~cm}^{-1}$; (c) $1724 \mathrm{~cm}^{-1} \mathrm{C}=\mathrm{O}$ stretch; (d) $1636 \mathrm{~cm}^{-1} \mathrm{C}=\mathrm{C}$ and Fermi overtone $1619 \mathrm{~cm}^{-1}$ from $\mathrm{C}=\mathrm{C}$ bend at $810 \mathrm{~cm}^{-1}$; (e) $1462 \mathrm{~cm}^{-1}$ and $1447 \mathrm{~cm}^{-1}$ CH2 stretch; (f) $1408 \mathrm{~cm}^{-1} \mathrm{O}-\mathrm{C}=\mathrm{O}$ stretch; (g) region between $1354 \mathrm{~cm}^{-1}$ and $1254 \mathrm{~cm}^{-1}$ typical C-N bands from PIP [21]; (h) $1181 \mathrm{~cm}^{-1}$ and $1158 \mathrm{~cm}^{-1} \mathrm{C}=\mathrm{O}$ stretch, shift is due to solidification; (i) $1010 \mathrm{~cm}^{-1}$ probably phosphor stretch from the photo initiator TPO; (j) $810 \mathrm{~cm}^{-1} \mathrm{C}=\mathrm{C}$ bend [22]. As can be seen, curing results in clearly observable spectral changes enabling monitoring of the curing process by FTIR spectroscopy. Absorption peaks attributed to the acrylic group, $\mathrm{f}$ and $\mathrm{j}$, expanded in insets, are suited for monitoring the curing process since they are well resolved and exhibit strong adsorptions that are clearly reduced upon curing. We selected the f peak, since this absorption peak was also used in a study of the effects of oxygen termination and inert conditions on curing by Studer et al. [18].

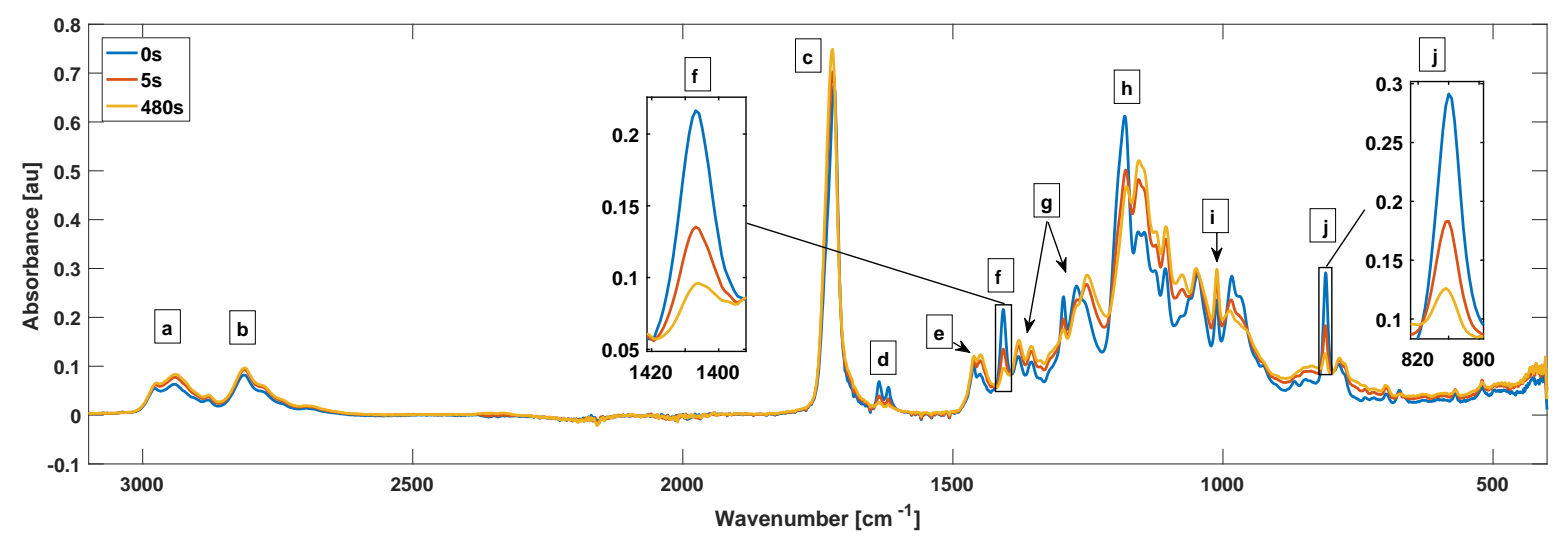

Figure 3. Fourier transform infrared (FTIR) of $300 \mu \mathrm{m}$ thick 1,3-AOBAE film mixed with $2.5 \mathrm{wt} \%$ diphenyl(2,4,6-trimethylbenzoyl) phosphine oxide (TPO) at different curing times 0,5 and $480 \mathrm{~s}$. $\mathrm{UV}$ cured in $\mathrm{N}_{2}$ with wavelength $365 \mathrm{~nm}$ and $11,9 \mathrm{~mW} / \mathrm{cm}^{2}$ of curing power. Peak $\mathrm{f}$, the $\mathrm{O}-\mathrm{C}=\mathrm{O}$ stretch at $1408 \mathrm{~cm}^{-1}$ was chosen for the monomer conversion rate calculation.
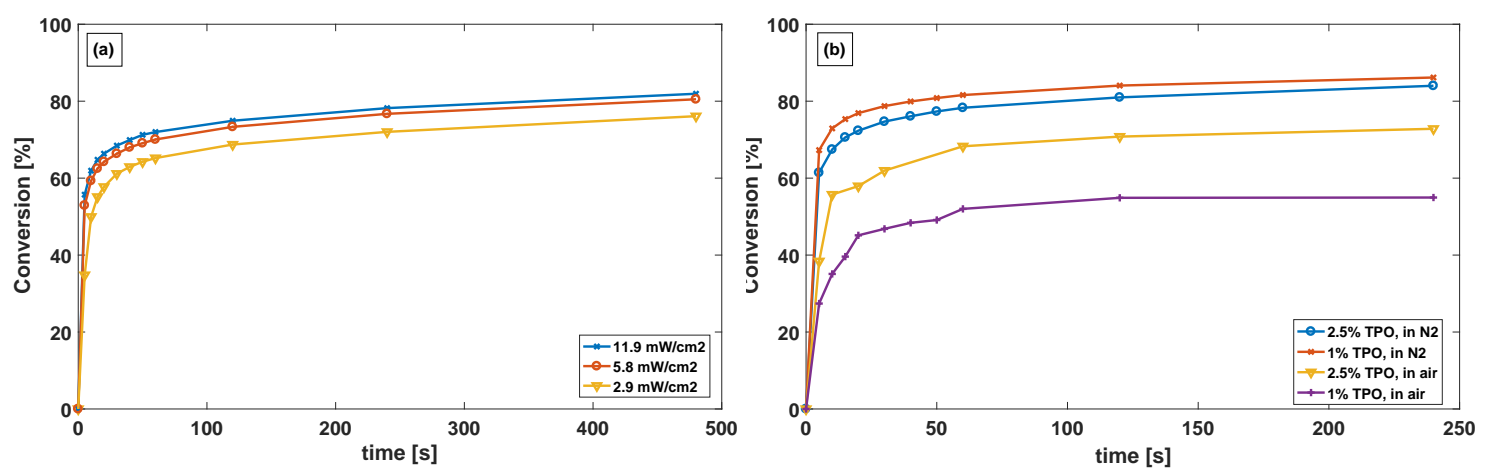

Figure 4. Monomer conversion of 1,3-AOBAE. In (a) monomer conversion of AOBAE films with $2.5 \mathrm{wt} \%$ TPO for different curing power $/ \mathrm{cm}^{2}$ in $\mathrm{N}_{2}$ atmosphere, $2.9,5.8$ and $11.9 \mathrm{~mW} / \mathrm{cm}^{2}$. (a) shows that monomer conversions for 5.8 and $11.9 \mathrm{~mW} / \mathrm{cm}^{2}$ are quite comparable and $2.9 \mathrm{~mW} / \mathrm{cm}^{2}$ a bit lower. In (b) the effect of TPO concentration, 2.5 and $1 \mathrm{wt} \%$, on the monomer conversion in AOBAE film is shown. This shows that while in $\mathrm{N}_{2}$ atmosphere, the difference is negligible, which is clearly different from when curing is carried out in air. 
The effect of different curing intensities and amount of TPO on the curing of the 1,3-AOBAE, can be seen in Figure 4. (a) curing in $\mathrm{N}_{2}$ atmosphere, shows that monomer conversions for 5.8 and $11.9 \mathrm{~mW} / \mathrm{cm}^{2}$ are quite analogous and $2.9 \mathrm{~mW} / \mathrm{cm}^{2}$ a bit lower, while (b) shows how the amount of TPO in the 1,3-AOBAE affects the curing in $\mathrm{N}_{2}$ and air for 1 and $2.5 \mathrm{wt} \%$ respectively. There is only a small difference in conversion between 1 and $2.5 \mathrm{wt} \%$ TPO in $\mathrm{N}_{2}$ atmosphere which indicates that $1 \mathrm{wt} \%$ is enough for the reaction. For conversion in air, the difference between 1 and $2.5 \mathrm{wt} \%$ is much greater, indicating that oxygen termination is more pronounced when curing is carried out in air atmosphere. This is consistent with observations made by Studer et al. [18,19].

\subsection{Cured 1,3-AOBAE Swell Response to $\mathrm{pH}$}

Figure 5 shows thickness measurements of AOBAE thin films that are exposed to different $\mathrm{pH}$ solutions. $0 \%$ on the Y-axis corresponds to the dry thickness state of the film, before any pH solution is added. As shown in Figure 5a, after a fast swelling rate during the first $5 \mathrm{~s}$, the final thickness for the films are reached after approximately $150 \mathrm{~s}$ but is also dependent on the $\mathrm{pH}$. The swelling rate during the first $5 \mathrm{~s}$ is approximately 3.0, 3.7 and $4.4 \%$, s, for $\mathrm{pH}$ 5.95, 5.70 and 5.53 respectively. Also seen in Figure $5 \mathrm{a}$ is that the response curve for $\mathrm{pH} 5.95$ only needs about $20 \mathrm{~s}$ to reach its final thickness, while up to $150 \mathrm{~s}$ are required for $\mathrm{pH} 5.53$ and 5.70. This indicates that the swelling is purely elastic for moderate swelling, while larger swelling requires rearranging of molecules. Similar results are observed also for thicker films, but with the main difference that a longer time is required for the film to reach its final thickness, see the blue curve (-*-) in Figure $5 \mathrm{~b}$. This is due to the diffusion process (time dependent), which is the main swelling mechanism in the hydrogel $[8,23]$. The orange curve $(-\nabla-)$ in Figure 5 b shows a $2.9 \mu \mathrm{m}$ film in $\mathrm{pH} 5.00$ that de-laminated from the substrate due to shear stress between the substrate and the film, observed after $30 \mathrm{~s}$ and approximately $30 \%$ swelling. Thicker films $(>3 \mu \mathrm{m})$ appeared to detach easier than thin films $(<3 \mu \mathrm{m})$, especially at lower $\mathrm{pH}(<\mathrm{pH} 6.5)$. This made swell experiments with thicker films difficult to conduct.
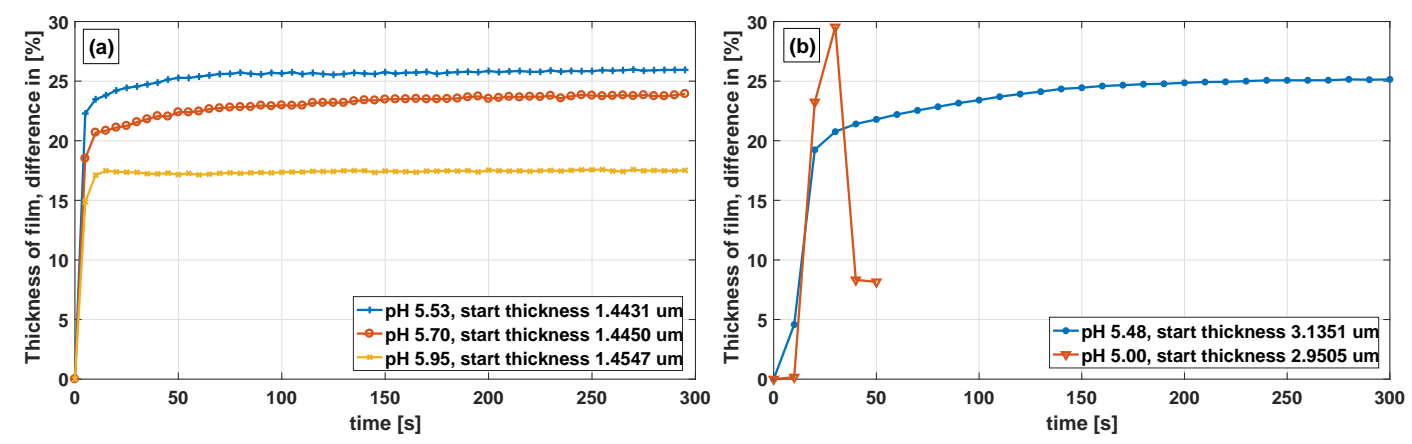

Figure 5. behavior of 1,3-OBAE mole ratio 2:1 that was exposed to different $\mathrm{pH}$ values. A value of $0 \%$ on the Y-axis equals the film's dry thickness before any $\mathrm{pH}$ solution is added. In (a) the response for a $1.4 \mu \mathrm{m}$ thick film in $\mathrm{pH} 5.53,5.70$ and 5.95 and in (b) the blue (-*-) curve shows the response of a $3.1 \mu \mathrm{m}$ thick film in $\mathrm{pH} 5.48$, where it can be seen that a thicker film is slower in its response to $\mathrm{pH}$ (compare with Figure 5a). The orange (- $\nabla-$ ) curve shows a $2.9 \mu \mathrm{m}$ thick film in $\mathrm{pH} 5.00$, which detached from the wafer surface after $30 \mathrm{~s}$.

\section{Discussion}

The experiments show that AOBAE can form $\mathrm{pH}$-responsive coatings. One objective was to manufacture AOBAEs that are fluid in the form of neat compounds at ambient temperature. A molecular design preventing crystallization by using asymmetric monomer units of the polymer was successfully explored.

In $\mathrm{pH}$-responsive coatings from cross-linked AOBAEs, swelling is driven by osmotic pressure induced by protonation of amino groups and is restricted by elastic force of the bonds forming cross-linked network [24]. Consequently, it can be expected that the swelling, which is a response to 
lowering the $\mathrm{pH}$, is dependent on the molecular weight of the AOBAE. Increasing the molecular weight of the AOBAE will increase the number density of amino groups and decrease the number density of cross-linking bonds in the cured coating, consequently increasing the swelling responsivity to $\mathrm{pH}$ changes. However, in devices where a hydrogel coating is immobilized on a device surface, swelling induces both adhesive and cohesive stress that may destroy the sensor structure [25]. By altering the mole ratio or the density of cross-linked acrylic groups, the sensitivity of the material is also altered. A higher concentration of amino groups increases the swelling and a higher density of cross-linked acrylic groups decreases the swelling [26,27]. Therefore, the responsive material needs to be optimized for the specific application where it is to be used.

The NMR spectra obtained from the AOBAEs, synthesized from 1,3-BDDA and PIP, were consistent with the proposed structure. The NMR spectrum of 1,3-AOBAE was compared with NMR-spectra previously recorded about PBAEs, based on 1,4-BDDA and PIP, cf. reference [12]. It can be noted that the additional peaks from the methyl group ( $g$ and $\left.g^{\prime}\right)$, as well as the NMR signals originating from the 1,3-butylene group, are broader. The 1,3-butylene group introduces asymmetry in two ways: by the chirality of the methine carbon and by the possibility of coupling to the either the side of the 1 or 3, "head and tail", positions of the molecule. The stereo and directional isomers are not assigned to specific spectral peaks in this study, but their presence can explain why the spectral peaks of the 1,3-butylene group exhibits a broad and more complicated pattern than the NMR-peaks of the symmetric 1,4-butylene group shown in reference [12]. More importantly, the isomerism present in 1,3-AOBEA prevents crystallization of the resulting monomers, which is in line with the intention of this work.

Surface priming with a molecule having an acrylic group covalently tethered to the substrate resulted in the samples with the best adhesion for swelling/de-swelling experiments. Depending on the surface to be coated (material and structure), a different surface priming might be needed. Adhesion of the coating requires priming the substrate with polymerize-able groups forming covalent bonds between the substrate and the $\mathrm{pH}$ responsive coating. This will reduce the risk for the film to detach from the substrate due to the physical forces from swelling. Apparently, the acrylic groups of the priming agent partake in the polymerization under curing. Further work in this class of sensor material coating should be aimed at providing coatings with improved stability for adhesion and swelling.

The curing conditions are crucial for a successful creation of a $\mathrm{pH}$-responsive coating. Curing by photo-initiated radical polymerization of the terminal groups proceeds best under inert atmosphere and agrees with previous reports from Studer et al. [18,19]. In Figure $4 \mathrm{a}$, the monomer conversion in $\mathrm{N}_{2}$ for 5.8 and $11.9 \mathrm{~mW} / \mathrm{cm}^{2}$ are very comparable, while slightly lower for $2.9 \mathrm{~mW} / \mathrm{cm}^{2}$. This can be explained by a lower rate of photolytic generation of radicals from the photo initiator and possibly termination of radicals by oxygen that may be present even after flushing with nitrogen gas. Our experiments also showed that a sufficient amount of photo initiator is necessary, especially for curing in air, to overcome the inhibitory effect of oxygen. This is shown in Figure $4 b$, where only a small difference in conversion is observed between 1 and $2.5 \%$ TPO in $\mathrm{N}_{2}$ atmosphere. A larger difference is observed for conversion in air, indicating that free radicals released from the TPO is reacting with oxygen at a fast enough rate to limit the conversion. Thus, we believe that in air atmosphere the rate of photolytic generation of radicals from the photo initiator is not fast enough to overcome the rate of new oxygen that is diffusing into the polymer from the atmosphere. There is a number of factors that influence the polymerization, including curing power, the thickness of the polymer film, the temperature and the oxygen levels in the atmosphere $[18,19]$, which should be taken into account and furthered studied.

From the swell tests on a flat surface, we have seen that the film detaches at lower $\mathrm{pH}$ due to the physical forces induced by the swelling. Though we believe that when coated on a round surface, such as an optical fiber, this problem will be much smaller. This because the polymer swells in all directions (the $\mathrm{x}, \mathrm{y}$ and $\mathrm{z}$ direction) and therefore also presses toward the fiber. Figure 5 a shows that the response curve of the three measurements differ. Possible explanation for this could be that for small degrees of swelling, swelling is fast and the gel network purely elastic, while at larger degrees of 
swelling, rearrangement of the gel network may be required and rate limiting, although this needs to be studied further for clarification. In Figure 5a the yellow (-x-) curve for $\mathrm{pH} 5.95$, we can also see that the thickness is constant over time. This indicates that the polymerization is complete and that no AOBAE material is dissolving out into the $\mathrm{pH}$-buffer. For thicker films, the response time is longer, which is shown in Figure 5 b blue $(-*-)$ curve, this is in agreement with Tanaka and Fillmore [23], who stated that the time constant $\tau$ for a spherical hydrogel has an $r^{2}$ relation $\tau=r^{2} / D_{\text {coop }}$. Where $\mathrm{r}$ is the radius of the sphere and $D_{\text {coop }}$ is the cooperative diffusion coefficient and also in agreement with De et al. [28], who concluded that the swelling kinetics of hydrogels is a diffusion limited process. The 1,3-AOBAE synthesized from a molar ratio of 2:1 has a swell difference of 9\%-units between $\mathrm{pH} 5.95$ and 5.53. Due to film detachment from the Si-wafer, measurements with lower $\mathrm{pH}$ than approximately 5.5 could not be made. This swell response to $\mathrm{pH}$ might seem small, although this can still induce a large difference in the effective permittivity and RI of the material. Permittivity and RI are physical attributes commonly used in electronic and optical sensing and thus this needs to be studied further.

\section{Conclusions}

A class of $\mathrm{pH}$ sensitive acrylic terminated OBAE has been studied with potential use as a $\mathrm{pH}$ responsive coating material in optical and printed electronics sensor systems. Batches of 1,3-AOBAE that in room temperature were liquids with various viscosity, were characterized with NMR and FTIR. FTIR was also used to monitor the curing behavior. Application of $\mathrm{pH}$ droplets on the responsive coating material with $\mathrm{pH} 5.53$ and $\mathrm{pH} 5.95$ resulted in a thickness difference with up to $9 \%$-units. The result is encouraging, demonstrating the potential use for the AOBAE as a pH-responsive coating on e.g., optical- or printed sensors. Swelling due to water uptake in a material also alter the materials other physical properties, for example permittivity and RI, parameters commonly used in sensor applications. The requirement of different sensor parameters (e.g., pH sensing range and accuracy) is to a large extent application dependent. However, these parameters are not solely determined by the responsive coating material, but also on the sensor design. Application of the AOBAE responsive coating material, both on an optical- and a printed sensors including design and characterization will be presented elsewhere.

Acknowledgments: The Knowledge Foundation (KKS), the Interreg Sweden-Norway Program and Fiber Optic Valley are gratefully acknowledged for the financial support. Mats Sandberg's work was financed by The Swedish Foundation for Strategic Environmental Research (Mistra) in the research program Mistra TerraClean. We are grateful for the help with NMR spectroscopy from Natalia Sjöberg.

Author Contributions: All authors conceived and designed the experiments. Krister Hammarling performed the experiments and analysed the FTIR and swell test data. Mats Sandberg provided advice on molecular design, curing conditions and interpretation of NMR spectra. Krister Hammarling wrote the manuscript, with the exception of the part about NMR, which was written by Mats Sandberg. All authors have proofread the manuscript.

Conflicts of Interest: The authors declare no conflict of interest. The founding sponsors had no role in the design of the study, in the collection, analyses, or interpretation of data, in the writing of the manuscript and in the decision to publish the results.

\section{Abbreviations}

The following abbreviations are used in this manuscript:

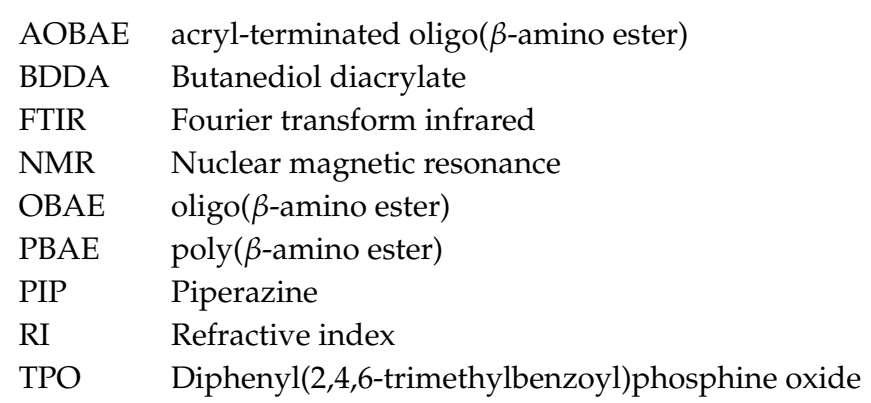




\section{References}

1. Sokolov, A.N.; Roberts, M.E.; Bao, Z. Fabrication of low-cost electronic biosensors. Mater. Today 2009, 12, 12-20.

2. Hart, J.K.; Martinez, K. Environmental sensor networks: A revolution in the earth system science? Earth-Sci. Rev. 2006, 78, 177-191.

3. Duarte, K.; Justino, C.I.; Freitas, A.C.; Gomes, A.M.; Duarte, A.C.; Rocha-Santos, T.A. Disposable sensors for environmental monitoring of lead, cadmium and mercury. TrAC Trends Anal. Chem. 2015, 64, 183-190.

4. Vanderroost, M.; Ragaert, P.; Devlieghere, F.; De Meulenaer, B. Intelligent food packaging: The next generation. Trends Food Sci. Technol. 2014, 39, 47-62.

5. de Vargas Sansalvador, I.M.P.; Fay, C.D.; Cleary, J.; Nightingale, A.M.; Mowlem, M.C.; Diamond, D. Autonomous reagent-based microfluidic pH sensor platform. Sens. Actuators B Chem. 2016, 225, 369-376.

6. Ganesh, A.B.; Radhakrishnan, T. Fiber-optic pH sensor. Fiber Integr. Opt. 2006, 25, 403-409.

7. Tan, C.; Ding, K.; Jin, B.; Seyfried, W.E.; Chen, Y. Development of an in situ pH calibrator in deep sea environments. IEEE/ASME Trans. Mechatron. 2012, 17, 8-15.

8. Richter, A.; Paschew, G.; Klatt, S.; Lienig, J.; Arndt, K.F.; Adler, H.J.P. Review on hydrogel-based pH sensors and microsensors. Sensors 2008, 8, 561-581.

9. Krebs, F.C. Fabrication and processing of polymer solar cells: A review of printing and coating techniques. Sol. Energy Mater. Sol. Cells 2009, 93, 394-412.

10. Lynn, D.M.; Langer, R. Degradable poly( $\beta$-amino esters): Synthesis, characterization, and self-assembly with plasmid DNA. J. Am. Chem. Soc. 2000, 122, 10761-10768.

11. Wu, D.; Liu, Y.; Jiang, X.; He, C.; Goh, S.H.; Leong, K.W. Hyperbranched poly(amino ester)s with different terminal amine groups for DNA delivery. Biomacromolecules 2006, 7, 1879-1883.

12. Kim, M.S.; Lee, D.S.; Choi, E.K.; Park, H.J.; Kim, J.S. Modulation of poly( $\beta$-amino ester) pH-sensitive polymers by molecular weight control. Macromol. Res. 2005, 13, 147-151.

13. Flory, P.J.; Leutner, F.S. Occurrence of head-to-head arrangements of structural units in polyvinyl alcohol. Chin. J. Polym. Sci. 1948, 3, 880-890.

14. Pascui, O.F.; Lohwasser, R.; Sommer, M.; Thelakkat, M.; Thurn-Albrecht, T.; Saalwächter, K. High crystallinity and nature of crystal- crystal phase transformations in regioregular poly(3-hexylthiophene). Macromolecules 2010, 43, 9401-9410.

15. Boltshauser, T.; Leme, C.A.; Baltes, H. High sensitivity CMOS humidity sensors with on-chip absolute capacitance measurement system. Sens. Actuators B Chem. 1993, 15, 75-80.

16. Chung, T.S. A critical review of polybenzimidazoles: Historical development and future R\&D. J. Macromol. Sci. Polym. Rev. 1997, 37, 277-301.

17. Dübendorfer, J.; Kunz, R.; Jobst, G.; Moser, I.; Urban, G. Integrated optical pH sensor using replicated chirped grating coupler sensor chips. Sens. Actuators B Chem. 1998, 50, 210-219.

18. Studer, K.; Decker, C.; Beck, E.; Schwalm, R. Overcoming oxygen inhibition in UV-curing of acrylate coatings by carbon dioxide inerting, Part I. Prog. Org. Coat. 2003, 48, 92-100.

19. Studer, K.; Decker, C.; Beck, E.; Schwalm, R. Overcoming oxygen inhibition in UV-curing of acrylate coatings by carbon dioxide inerting: Part II. Prog. Org. Coat. 2003, 48, 101-111.

20. Britton, H.T.S.; Robinson, R.A. CXCVIII.-Universal buffer solutions and the dissociation constant of veronal. J. Chem. Soc. (Resumed) 1931, 1456-1462, doi:10.1039/JR9310001456.

21. Helaly, F.M.; Hashem, M.S. Preparation and characterization of poly( $\beta$-amino ester) capsules for slow release of bioactive material. J. Encapsulation Adsorpt. Sci. 2013, 3, 65.

22. Silverstein, R.M.; Webster, F.X.; Kiemle, D.J.; Bryce, D.L. Spectrometric Identification of Organic Compounds; John Wiley \& Sons: New York, NY, USA, 2014.

23. Tanaka, T.; Fillmore, D.J. Kinetics of swelling of gels. J. Chem. Phys. 1979, 70, 1214-1218.

24. Siegel, R.A.; Firestone, B.A. pH-dependent equilibrium swelling properties of hydrophobic polyelectrolyte copolymer gels. Macromolecules 1988, 21, 3254-3259.

25. Doi, M. Gel dynamics. J. Phys. Soc. Jpn. 2009, 78, doi:10.1143/jpsj.78.052001.

26. Peppas, N.A.; Leobandung, W. Stimuli-sensitive hydrogels: Ideal carriers for chronobiology and chronotherapy. J. Biomater. Sci., Polym. Ed. 2004, 15, 125-144. 
27. Çaykara, T.; Turan, E. Effect of the amount and type of the crosslinker on the swelling behavior of temperature-sensitive poly(N-tert-butylacrylamide-co-acrylamide) hydrogels. Colloid. Polym. Sci. 2006, 284, 1038-1048.

28. De, S.K.; Aluru, N.; Johnson, B.; Crone, W.; Beebe, D.J.; Moore, J. Equilibrium swelling and kinetics of pH-responsive hydrogels: Models, experiments, and simulations. J. Microelectromech. Syst. 2002, $11,544-555$.

(C) 2018 by the authors. Licensee MDPI, Basel, Switzerland. This article is an open access article distributed under the terms and conditions of the Creative Commons Attribution (CC BY) license (http://creativecommons.org/licenses/by/4.0/). 